\title{
OPEN Microplastics pollution in salt pans from the Maheshkhali Channel, Bangladesh
}

\author{
Md. Refat Jahan Rakib ${ }^{1 凶}$, Sultan Al Nahian ${ }^{2}$, María B. Alfonso ${ }^{3}$, Mayeen Uddin Khandaker ${ }^{4}$, \\ Christian Ebere Enyoh ${ }^{5}$, Fauziah Shahul Hamid ${ }^{6,7}$, Abdullah Alsubaie $^{8}$, \\ Abdulraheem S. A. Almalki ${ }^{9}$, D. A. Bradley ${ }^{4,10}$, Hamidreza Mohafez ${ }^{11 \bowtie}$ \& \\ Mohammad Aminul Islam ${ }^{12}$
}

Microplastics (MP) were recognized as an emergent pollution problem due to their ubiquitous nature and bioaccumulative potential. Those present in salt for consumption could represent a human exposure route through dietary uptake. The current study, conducted in Bangladesh, reports microplastics contamination in coarse salt prepared for human consumption. Sea salt samples were collected from eight representative salt pans located in the country's largest salt farming area, in the Maheshkhali Channel, along the Bay of Bengal. Microplastics were detected in all samples, with mean concentrations ranging from $78 \pm 9.33$ to $137 \pm 21.70$ particles $\mathrm{kg}^{-1}$, mostly white and ranging in size from $500-1000 \mu \mathrm{m}$. The prevalent types were: fragments $(48 \%)>$ films $(22 \%)>$ fibers $(15 \%)>$ granules and lines (both 9\%). Fourier transform mid-IR and near-IR spectra (FT-MIR-NIR) analysis registered terephthalate $(48 \%)$, polypropylene $(20 \%)$, polyethylene $(17 \%)$, and polystyrene $(15 \%)$ in all samples. These results contribute to the MP's pollution knowledge in sea salts to understand and reduce this significant human exposure route and environmental pollution source in the future.

Plastic pollution in the marine environment has become a major global problem ${ }^{1}$. The degradation of the plastic waste in the seawater is not the end of the problem as the microplastics (MP) generated have a destructive potential for the environment. Added to this is the release of chemical compounds, further worsening the damage to the environment ${ }^{2}$. Furthermore, most plastics in the environment endure for a very long time ${ }^{3}$. According to the G20 Implementation Framework for Actions on Marine Plastic Litter, 20 countries are responsible for $80 \%$ of the plastic debris found in the sea, from which $90 \%$ originate from land-based sources. According to a study by Jambeck et al. $^{4}$, the significant percentage of the world's ocean plastics pollution is from Asia, with China contributing $28 \%$ of the mismanaged plastic waste, followed by Indonesia (10\%), the Philippines and Vietnam (both 6\%), Thailand 3.2\%, Egypt 3\%, Nigeria 2.7\%, and South Africa $2 \%$.

MPs become more threatening than large plastic materials because they could be swallowed and concentrated by aquatic organisms, including plankton ${ }^{5,6}$. A lower limit to MP size study has not yet been defined, although most investigations have focused on the $0.3-5 \mathrm{~mm}$ size range ${ }^{7}$. MP can be classified into two basic groups, namely primary and secondary, depending on their origin. Primary MP are industry-made particles, mainly used in

\footnotetext{
${ }^{1}$ Department of Fisheries and Marine Science, Faculty of Science, Noakhali Science and Technology University, Noakhali, Bangladesh. ${ }^{2}$ Bangladesh Oceanographic Research Institute, Cox's Bazar, Bangladesh. ${ }^{3}$ Instituto Argentino de Oceanografía (IADO-CONICET-UNS), Florida 8000, B8000BFW Bahía Blanca, Argentina. ${ }^{4}$ Centre for Applied Physics and Radiation Technologies, School of Engineering and Technology, Sunway University, 47500 Bandar Sunway, Selangor, Malaysia. ${ }^{5}$ Group Research in Analytical Chemistry, Environment and Climate Change (GRACE \& CC), Department of Chemistry, Faculty of Science, Imo State University Owerri, P. M. B 2000, Imo State, Nigeria. ${ }^{6}$ Institute of Biological Sciences, Faculty of Science, University of Malaya, 50603 Kuala Lumpur, Malaysia. ${ }^{7}$ Center for Research in Waste Management, Faculty of Science, University of Malaya, 50603 Kuala Lumpur, Malaysia. ${ }^{8}$ Department of Physics, College of Khurma, Taif University, P.O. Box 11099, Taif 21944, Saudi Arabia. ${ }^{9}$ Department of Chemistry, Faculty of Science, Taif University, Taif 21974, Saudi Arabia. ${ }^{10}$ Department of Physics, University of Surrey, Guildford GU2 7XH, UK. ${ }^{11}$ Department of Biomedical Engineering, Faculty of Engineering, Universiti Malaya, 50603 Kuala Lumpur, Malaysia. ${ }^{12}$ Department of Electrical Engineering, Faculty of Engineering, Universiti Malaya, 50603 Kuala Lumpur, Malaysia. ${ }^{\circledR e m a i l: ~ r i f a t j a h a n r a k i b @ g m a i l . c o m ; ~ h . m o h a f e z @ ~}$ um.edu.my
} 


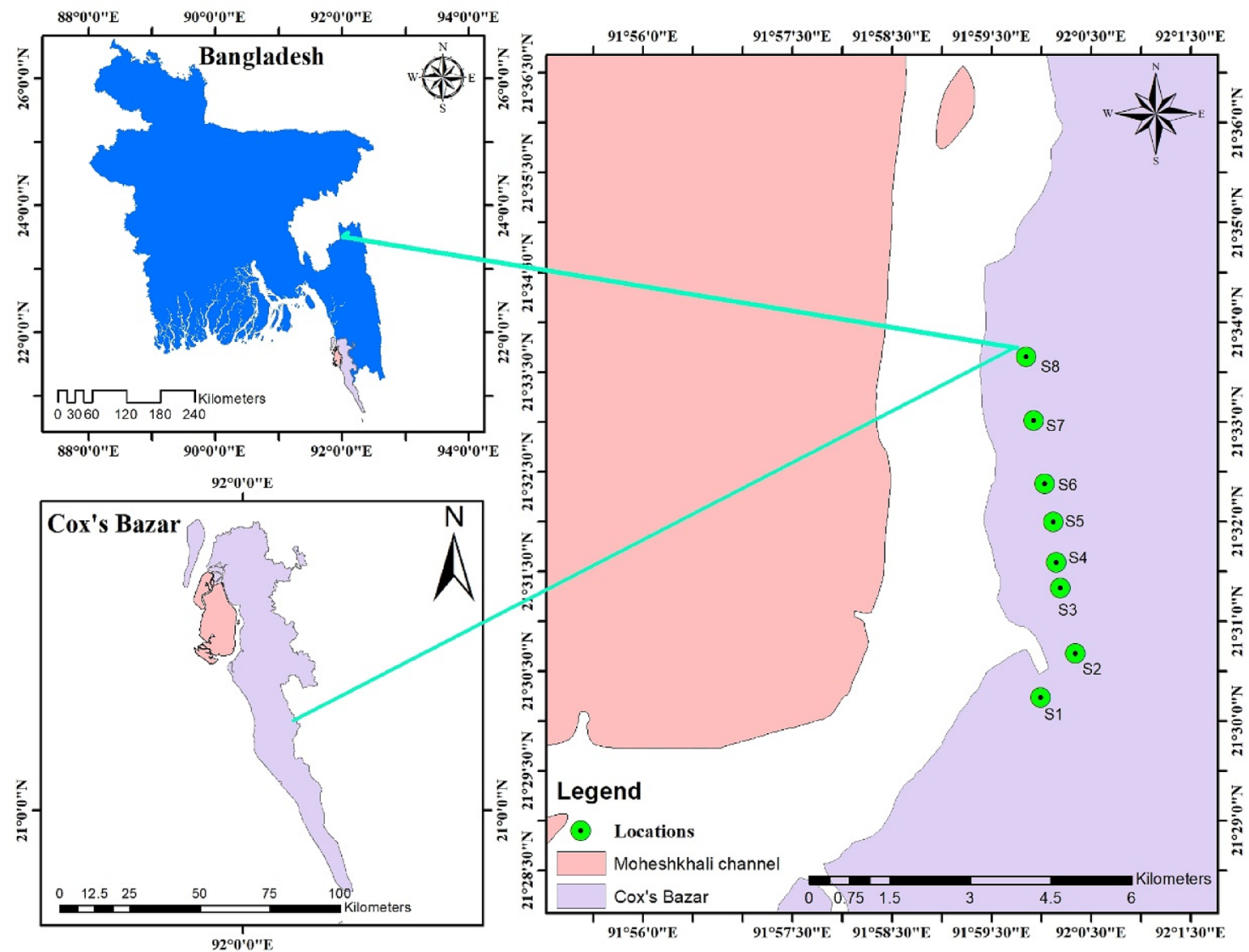

Figure 1. The geographic location of the sampling points along the Maheshkhali Channel coast. This map was constructed using ArcGIS 10.7.

commercial formulations, from cosmetics and toothpaste to micro-additives in synthetic paints and coatings ${ }^{8}$. Secondary MP results from the fragmentation of larger plastics ${ }^{8}$.

When it comes to MP pollution, salts for consumption are not exempt from the problem. Table salts are obtained from mining mineral rock or the evaporation of water sources at sea ${ }^{1}$. During the production process, saltwater undergoes different physical processes. It is first pumped into evaporation ponds, subsequently concentrated and crystallized by the action of the sun and wind, then being cut and packed for sale ${ }^{9}$. Accordingly, the final product may contain concentrates of the anthropogenic contaminants already present in the saltwater ${ }^{10}$.

Sea salt is highly commercialized, being consumed worldwide, with the number of studies focusing on MP pollution in table salts increasing ${ }^{11,12}$. Around 60 salt processing mills exist in the Cox's Bazar district in Bangladesh $^{9}$, wherein washing, crushing, iodine mixing, drying, and packing are carried out. About $25 \%$ of the raw salt is transformed as waste during processing, while the remaining $75 \%$ is crushed and packed as iodide salt and distributed throughout the country, predominantly for human consumption. Despite the high consumption rates, there has been a lack of data on the presence of MP in table salt from Bangladesh. Therefore the present study seeks to analyze for the first time the abundance, characteristics, and polymer composition of MP pollution in commercial salts obtained from salt pans along the Bangladesh coast. It is expected that this study will form a baseline for MP salt pollution for the country, also enhancing knowledge about this emergent pollutant issue.

\section{Material and methods}

Study area. Located at the head of the Bay of Bengal, the coastline of Bangladesh represents a diversified hydro-geo-morphological environment, with several interruptions by estuarine inlets ${ }^{13}$. The present study was performed in the Maheshkhali Channel (MC), located on the southeast coast of the Bay of Bengal (Fig. 1). The channel includes the Bakkhali River to the south, discharging domestic, agricultural, and industrial waste before falling into the Bay ${ }^{14,15}$. This area has a semidiurnal tidal regime, with sea currents increasing from south to north, mainly near the coastal area ${ }^{16}$. Its hydrology is also heavily influenced by the monsoon season (JuneSeptember $)^{9}$. The surrounding area to MC is highly urbanized, including Chittagong in the north, forming the second-largest city in Bangladesh (approaching 8,440,000 inhabitants) and Cox's Bazar beach at the east (with 85,000 daily visitors) (Fig. 1), the longest natural beach in the world with many hotels, a fishing industry, tourism and unplanned urbanization (e.g., a camp for displaced Rohingya refugees) ${ }^{9}$.

The MC is one of the most popular places in Bangladesh for salt production ${ }^{9}$. Extended mud flat shores are present along its coast, where most salt pans have been established ${ }^{9}$. The post-monsoon season (November to April) gives rise to favorable meteorological conditions for salt production, farmers selling the raw salt directly 

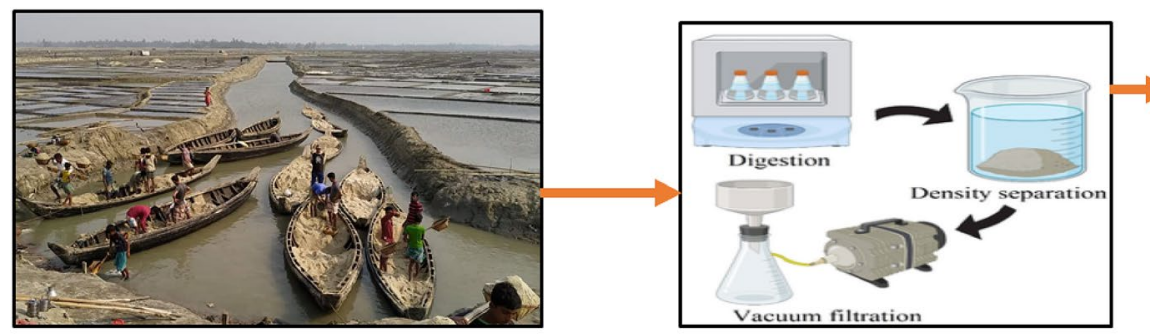

Salts Pans Sample collection

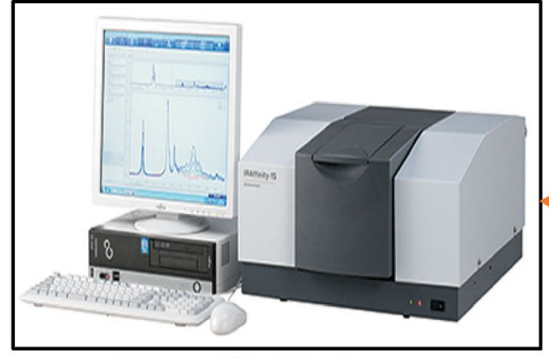

Polymer Identification
Extraction

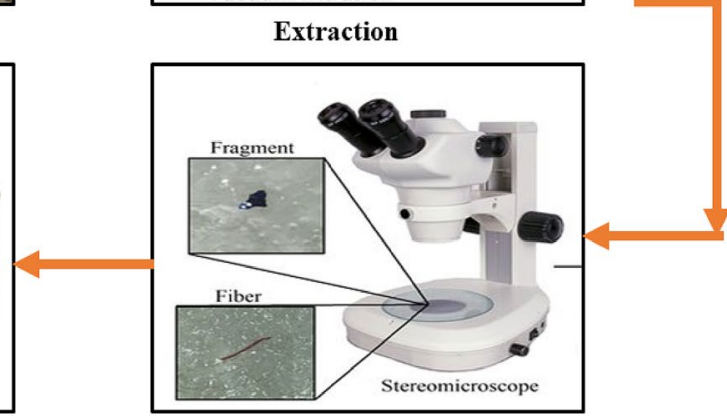

Microscope

Figure 2. Procedural steps for microplastics analysis in sea salts samples. This diagram was constructed using PowerPoint 2016.

to traders ${ }^{9}$. Besides salt pans and processing industries, other productive activities developed in this area are traditional fisheries, commercial shrimp farms, a fishing port, fish processing industries, ship and boat making factories, and paint industries. Therefore, MC is seriously impacted by chemical effluents and waste effluents from many unplanned industries ${ }^{14}$.

Samples collection. A total of eight representative salt pans (large in terms of relative size or hosting the greatest numbers of salt pans in a particular location) were selected to collect the samples for this study. Unrefined sea salt for consumption samples were collected from the selected natural salt pans along the southeast coast of MC (S1-S8) (Fig. 1). Sampling was performed between August (2020) and September (2021) (the postmonsoon period). Approximately $500 \mathrm{~g}$ of salts were collected using a metal spoon at each site, placed in a clean labeled $1 \mathrm{~L}$ glass bottle, and transported to the laboratory.

Samples digestion and treatment. Each sample was divided into three $(n=3)$ subsamples for further analyses. Then $100 \mathrm{~mL}$ of $30 \% \mathrm{H}_{2} \mathrm{O}_{2}$ were added to each sample in cleaned glass flasks and heated in a bath held at $65^{\circ} \mathrm{C}$ for $24 \mathrm{~h}$ to digest any organic matter (Fig. 2). Approximately $1000 \mathrm{~mL}$ of filtered water was added to each bottle, and a glass rod was used to stir the salts until fully dissolved. The obtained salt solution was immediately filtered with cellulose nitrate filter paper ( $47 \mathrm{~mm}$ diameter, $0.45 \mu \mathrm{m}$ pore size) using a vacuum system. The filter was then placed into a clean glass Petri dish and dried at room temperature.

Quality control. All liquids were filtered using $0.45 \mu \mathrm{m}$ pore size filter paper before use to avoid MP contamination. Also, all the glass material was rinsed three times with purified water. The samples were kept covered when they were not under analysis. Lab staff used cotton lab coats and nitrile gloves to avoid any source of extraneous plastic contamination. Three blank samples without salt were analyzed simultaneously to correct for any possible MP contamination from sample processing. No MP were found in blank samples.

Visual observation and polymer FT-MIR-NIR analysis. The filters were visually inspected using an Olympus SZX16 stereomicroscope, and images were taken. Visual assessment was performed in order to identify the shape and color of MP. Then, particles were randomly selected from each site (approximately $30 \%$ ) for Fourier Transform Mid- and Near-Infra Red (FT-MIR-NIR) polymer analysis using a Perkin Elmer FTMIR-NIR system. MP abundance was calculated based on visual observation and FT-MIR-NIR plastic polymer confirmation. For FT-MIR-NIR analysis, the spectrum range was set to $4000-675 \mathrm{~cm}^{-1}$ with a $3 \mathrm{~s}$ and $8 \mathrm{~cm}^{-1}$ resolution collection for all samples. All the spectra were then compared with the spectra library to identify the polymer type.

Data analysis and risks assessment. Statistical analysis was performed using SPSS Version $20\left(\mathrm{IBM}^{\oplus}\right)$. Results were reported as mean values and standard deviation from replicates. Due to the non-normal distribution of data, the Kruskal-Wallis test followed by pairwise Dunn's tests were performed to determine any differences in MP concentrations among sampling sites ${ }^{17}$. Results were presented as boxplot and p-values. Statistical significance was set at $\mathrm{p}<0.05$. 


\begin{tabular}{|c|c|c|c|c|c|c|c|c|}
\hline & S1 & S2 & S3 & S4 & S5 & S6 & S7 & S8 \\
\hline \multicolumn{9}{|l|}{ Shape } \\
\hline Pellet & $6.3 \pm 2.1$ & $8.7 \pm 3.5$ & $10.7 \pm 2.3$ & $8.0 \pm 2.0$ & $7.0 \pm 3.0$ & $12.7 \pm 4.0$ & $8.7 \pm 2.1$ & $9.3 \pm 6.4$ \\
\hline Fiber & $5.3 \pm 1.5$ & $8.7 \pm 3.0$ & $14.0 \pm 4.6$ & $18.3 \pm 3.0$ & $12.3 \pm 3.5$ & $18.0 \pm 3.6$ & $26.3 \pm 5.0$ & $19.7 \pm 5.7$ \\
\hline Fragment & $37.7 \pm 3.5$ & $43.7 \pm 9.1$ & $27.0 \pm 9.2$ & $42.7 \pm 10.5$ & $32.7 \pm 4.7$ & $51.3 \pm 13.6$ & $59.3 \pm 16.9$ & $65.3 \pm 7.5$ \\
\hline Film & $20.3 \pm 6.1$ & $13.7 \pm 6.6$ & $15.7 \pm 5.5$ & $22.7 \pm 5.7$ & $39.3 \pm 7.5$ & $22.0 \pm 3.6$ & $30.3 \pm 5.5$ & $17.7 \pm 6.1$ \\
\hline Line & $7.3 \pm 3.5$ & $4.0 \pm 2.0$ & $7.3 \pm 1.5$ & $11.0 \pm 2.0$ & $17.0 \pm 5.3$ & $5.7 \pm 2.5$ & $12.0 \pm 5.0$ & $7.0 \pm 2.6$ \\
\hline \multicolumn{9}{|l|}{ Color } \\
\hline White & $8.0 \pm 3.6$ & $10.3 \pm 2.5$ & $7.3 \pm 3.1$ & $10.7 \pm 1.5$ & $9.7 \pm 2.1$ & $11.3 \pm 5.0$ & $19.7 \pm 4.2$ & $14.0 \pm 5.3$ \\
\hline Blue & $2.3 \pm 0.6$ & $3.0 \pm 1.0$ & $6.3 \pm 1.5$ & $5.3 \pm 2.5$ & $4.0 \pm 2.0$ & $6.3 \pm 1.5$ & $5.7 \pm 1.5$ & $4.3 \pm 2.5$ \\
\hline Green & $3.3 \pm 0.6$ & $1.7 \pm 0.6$ & $2.3 \pm 2.1$ & $3.0 \pm 2.6$ & $3.3 \pm 1.5$ & $5.3 \pm 4.2$ & $3.7 \pm 2.5$ & $2.7 \pm 1.2$ \\
\hline Black & $4.3 \pm 1.5$ & $4.3 \pm 1.5$ & $3.0 \pm 1.7$ & $5.0 \pm 2.6$ & $5.7 \pm 3.1$ & $3.0 \pm 2.0$ & $7.0 \pm 5.2$ & $9.0 \pm 3.6$ \\
\hline Pink & $1.7 \pm 0.6$ & $1.0 \pm 1.0$ & $0.7 \pm 0.6$ & $4.0 \pm 1.0$ & $2.7 \pm 1.5$ & $2.0 \pm 1.0$ & $1.3 \pm 0.6$ & $1.0 \pm 1.0$ \\
\hline Transparent & $2.3 \pm 0.6$ & $2.7 \pm 0.1$ & $2.3 \pm 0.6$ & $1.7 \pm 1.5$ & $3.7 \pm 3.1$ & $3.0 \pm 1.0$ & $3.3 \pm 1.5$ & $4.7 \pm 2.1$ \\
\hline Colorless & $1.3 \pm 0.6$ & $2.0 \pm 1.0$ & $0.7 \pm 0.6$ & $1.0 \pm 1.0$ & $2.7 \pm 0.6$ & $3.7 \pm 1.5$ & $1.0 \pm 1.0$ & $1.7 \pm 0.6$ \\
\hline \multicolumn{9}{|c|}{ Size range $(\mu \mathrm{m})$} \\
\hline $250-500$ & $6.0 \pm 3.0$ & $7.0 \pm 2.6$ & $6.3 \pm 3.1$ & $8.7 \pm 4.7$ & $6.7 \pm 4.2$ & $9.0 \pm 2.6$ & $10.7 \pm 4.0$ & $9.0 \pm 2.6$ \\
\hline $1000-500$ & $9.7 \pm 2.5$ & $10.3 \pm 4.2$ & $7.0 \pm 2.6$ & $12.3 \pm 2.1$ & $13.0 \pm 4.0$ & $13.7 \pm 4.2$ & $17.3 \pm 5.5$ & $15.3 \pm 2.5$ \\
\hline $1000-5000$ & $7.7 \pm 3.1$ & $7.7 \pm 2.5$ & $9.3 \pm 3.1$ & $9.7 \pm 2.1$ & $11.0 \pm 7.2$ & $12.0 \pm 3.0$ & $13.7 \pm 5.7$ & $13.0 \pm 5.3$ \\
\hline \multicolumn{9}{|l|}{ Polymer type } \\
\hline $\mathrm{PP}$ & $7.0 \pm 4.4$ & $5.3 \pm 2.08$ & $5.3 \pm 2.1$ & $6.3 \pm 1.5$ & $7.7 \pm 3.2$ & $7.0 \pm 4.0$ & $9.3 \pm 3.1$ & $7.0 \pm 2.0$ \\
\hline PET & $10.7 \pm 4.2$ & $11.7 \pm 3.1$ & $10.0 \pm 6.2$ & $15.3 \pm 4.2$ & $17.3 \pm 4.9$ & $19.3 \pm 7.8$ & $22.0 \pm 6.6$ & $21.7 \pm 9.1$ \\
\hline PS & $4.7 \pm 1.5$ & $4.3 \pm 1.5$ & $5.0 \pm 4.6$ & $5.0 \pm 2.0$ & $5.0 \pm 4.4$ & $5.7 \pm 3.1$ & $5.7 \pm 3.8$ & $5.0 \pm 1.7$ \\
\hline $\mathrm{PE}$ & $3.7 \pm 2.1$ & $5.0 \pm 1.0$ & $4.7 \pm 1.5$ & $7.3 \pm 4.2$ & $6.3 \pm 6.7$ & $4.3 \pm 2.1$ & $8.7 \pm 3.1$ & $6.0 \pm 1.0$ \\
\hline Total & $77.0 \pm 11.1$ & $78.7 \pm 11.0$ & $74.7 \pm 11.9$ & $102.7 \pm 11.4$ & $108.3 \pm 14.6$ & $109.7 \pm 17.9$ & $136.7 \pm 23.5$ & $119.0 \pm 22.6$ \\
\hline
\end{tabular}

Table 1. Microplastic abundance (Particles $\mathrm{kg}^{-1}$ ) (Mean value \pm SD) by shape, color, size range and, polymer categories in unpacked coarse salt samples from stations $\mathrm{S} 1$ to $\mathrm{S} 8(\mathrm{n}=3)$.

$M P$ risks assessment. Besides the residual monomers, additional polymerization pollutants may be present in a plastic object. These include oligomers, low-atomic-weight polymer segments, catalyst residues, and polymerization solvents, as well as a broad range of plastic additives, such as handling aids and final product additives ${ }^{18}$. Because the majority of these non-polymeric segments have a low atomic weight, they could migrate from the MP item into the salt and then into humans, where they may cause damage. Therefore, it is crucial to estimate the risks of these polymers. The risks assessment of MP in the salt were estimated using the polymer risk indices $\left(\mathrm{pR}_{\mathrm{i}}\right)$ following the procedure detailed in previous work ${ }^{18}$, presented in Eqs. (1) and (2) as follows:

$$
\begin{gathered}
p R_{i}=\sum\left(\frac{p_{i}}{p_{T}} \times S_{j}\right) \\
p R_{\text {overall }}=\left(p R_{1} \times p R_{2} \times p R_{3} \times \ldots p R_{n}\right)^{1 / n}
\end{gathered}
$$

where $\mathrm{p}_{\mathrm{i}}$ is the number of each MP polymer observed in salt sample $\mathrm{i}$ and $\mathrm{p}_{\mathrm{T}}$ is the total number of different MP polymers identified in salt sample i. $S_{\mathrm{j}}$ is the chemical toxicity coefficient or risk score, given as Polyethylene (PE), 11; Polyethylene terephthalate (PET), 4; Polypropylene (PP), 1, and Polystyrene (PS), $30^{18}$. The polymeric risk indices (pRi) classification is as follows: low when $<150$, medium when 150-300, considerable when 300-600, high 600-1200, and very high when $>1200^{18}$. Due to the simplicity of the index, it could be applied to different types of environments, but at the same time, the conclusions from it are limited and should be considered with caution.

\section{Result and discussion}

MPs abundance. In Table $1 \mathrm{MP}$ abundance (mean value \pm standard deviation) values are presented by shape, size range, color and polymer type categories for each sampling site. MP were found in all analyzed salt samples including pellets, fibers, fragments, films and lines (Fig. 3). MP total abundance values per site ranged from 74.7 to 136.7 particles $\mathrm{kg}^{-1}$ in the following order of increasing abundance: $\mathrm{S} 3<\mathrm{S} 1<\mathrm{S} 2<\mathrm{S} 4<\mathrm{S} 5<\mathrm{S} 6<\mathrm{S} 8$ $<$ S7 (Fig. 4, Table 1). The measured values show significant difference among sites (Kruskal Wallis, $\mathrm{p}=0.013$ ). The Dunn's test results reveal significant differences, with S1, S2 and S3 differing from S7 ( $p=0.006 ; p=0.007$ and $\mathrm{p}=0.005$, respectively) and $\mathrm{S} 8(\mathrm{p}=0.023 ; \mathrm{p}=0.028$ and $\mathrm{p}=0.019$, respectively), $\mathrm{S} 3$ also being significantly different from S6 $(\mathrm{p}=0.05)$.

It is expected that MP in seawater was the primary source of contamination of the sea salts ${ }^{19-22}$. According to these results, MP contamination in the salt pans from the Maheshkhali Channel coast can be classified into three zones of increasing pollution: a lower zone (S1 to S3), an intermediate zone (S4 and S5), and a higher 

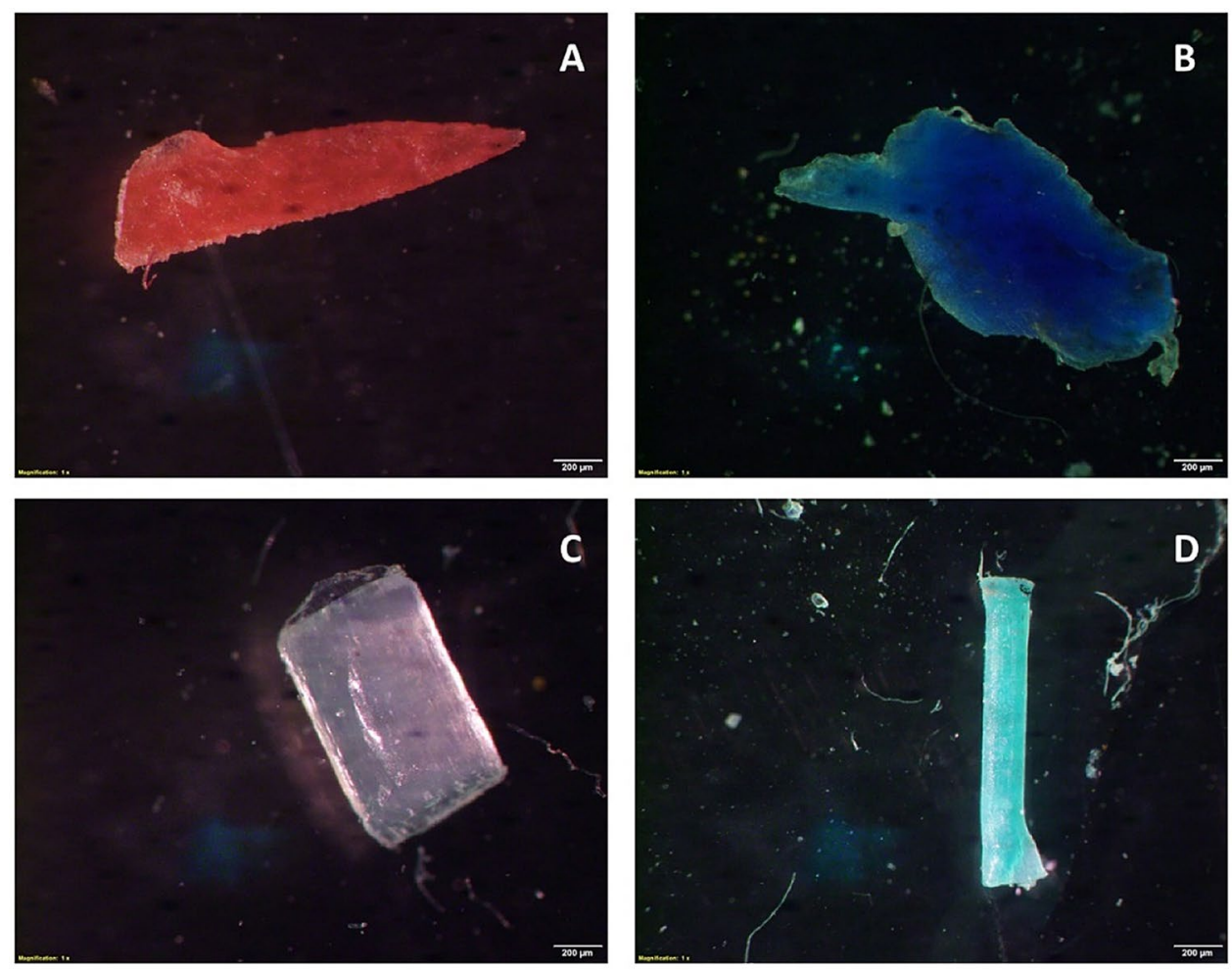

Figure 3. Photographs of different MP shapes found in salt samples: (a) red fragment; (b) blue fragment; (c) pellet; (d) line.

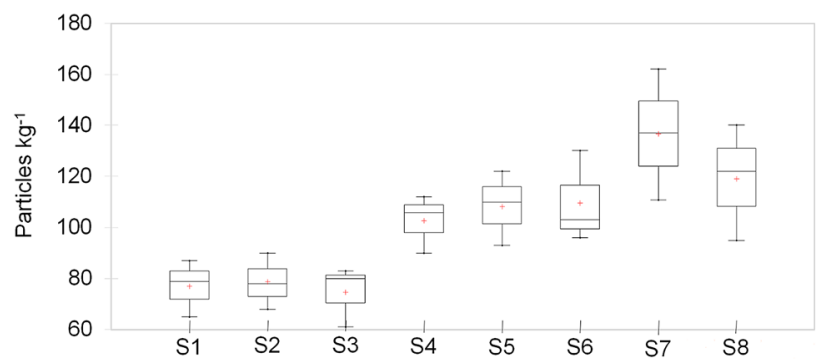

Figure 4. Box plot of MP abundance (particles $\mathrm{kg}^{-1}$ ) for the sampling sites S1 to S8.

zone (S6 to S8). A possible explanation for these values could be the different disposition of the study sites in the channel and the hydrology and current (rate of water movement) differences. According to Misra et al. ${ }^{16}$, seawater current flows with higher intensity from the south of the Bay of Bengal to the north. Therefore, any $\mathrm{MP}$ in seawater flowing from the Bay of Bengal inside MC (S1 to S8 direction) will probably accumulate in the inner part of the channel. However, the higher MP concentration in S7 with respect to S8 suggests that other environmental variables or anthropogenic sources could affect MP presence in MC, and more variables should be assessed in future studies to arrive at better conclusions. It should also be mentioned that the plastic film used in salt pans for desiccation is of high potential as a source of MP. Other possible MP sources could be plastic pollution from fishing, urbanization, and tourism activities in the surrounding area. Furthermore, runoff from the land and atmospheric fallout could also be potential contributory pathways ${ }^{23}$, not least given that Bangladesh is subject to the influence of the monsoon, with high rain values and salt production developing after the monsoon season ${ }^{9}$. Future research, including seawater and atmospheric MP samples, should be considered to confirm these assumptions.

Although there are no previous studies of MP abundance in salt samples, other studies have been conducted in the coastal zone of the Bay of Bengal adjacent to Bangladesh. A study conducted by Rahman et al. ${ }^{24}$ in beach sediments from Cox's Bazar beach registered relatively low MP values of $8.1 \pm 2.9$ particles $\mathrm{kg}^{-1} \mathrm{while}$, in a study of intertidal sediments from the same area by Hossain et al. ${ }^{25}$, higher MP values of $368.68 \pm 10.65$ particles kg $^{-1}$ were reported. Both studies attributed their spatial MP variation to be due to the tidal current, wave energy, beach orientation, river discharges, and human activity. Finally, another study in beach sediments found MP concentrations up to 1100 particles $\mathrm{kg}^{-1}$, attributing these high values to increasing urbanization and tourism ${ }^{26}$. 


\begin{tabular}{|c|c|c|c|c|c|}
\hline \multirow[b]{2}{*}{ Sea } & \multirow[b]{2}{*}{ Country } & \multicolumn{3}{|l|}{ MP characteristics } & \multirow[b]{2}{*}{ References } \\
\hline & & Polymer type & Particle size range $(\mu \mathrm{m})$ & $\begin{array}{l}\text { Concentration (Particles } \\
\mathrm{kg}^{-1} \text { ) }\end{array}$ & \\
\hline Bay of Bengal & Bangladesh & PP, PET, PS, PE & $250-5000$ & $78-137$ & This study \\
\hline \multirow{7}{*}{ Pacific Ocean } & Thailand & PE, PET, PP, PVC & $\mid 100-5000$ & $80-600$ & 12 \\
\hline & Vietnam & Acrylic, PE, PP, PU & $100-5000$ & $100-200$ & 12 \\
\hline & New Zealand & PE & $160-980$ & $0-1$ & 11 \\
\hline & South Korea & $\begin{array}{l}\text { Acrylic, Nylon, PE, } \\
\text { PET, PP }\end{array}$ & $100-3000$ & $100-300$ & 12 \\
\hline & Japan & PE, PET & $160-980$ & $0-1$ & 11 \\
\hline & \begin{tabular}{|l} 
Chinese Taipei \\
\end{tabular} & PE, PET, PP & $100-2000$ & $0-1300$ & 12 \\
\hline & Australia & $\begin{array}{l}\text { Acrylic, Nylon, PE, PET, } \\
\text { PP, PS }\end{array}$ & $100-3000$ & 80 & 12 \\
\hline \multirow{5}{*}{ Mediterranean Sea } & Turkey & PE, PET, PP, PU, PA, PVC & $<100$ & $18-84$ & 28 \\
\hline & Spain & PE, PET, PP & $30-3500$ & $80-280$ & 29 \\
\hline & Italy & PE, PP & $4-2100$ & $22-594$ & 27 \\
\hline & France & PE, PET, PP & $160-980$ & $0-2$ & 11 \\
\hline & Croatia & PE, PP & $15-4628$ & \begin{tabular}{|l|}
$13,500-19,800$ \\
\end{tabular} & 27 \\
\hline \multirow{2}{*}{ Indic Ocean } & India & Nylon, PE, PET, PP, PVC & $50-600$ & $100-5000$ & 12 \\
\hline & Malaysia & PP & $160-980$ & $0-1$ & 11 \\
\hline Black Sea & Bulgaria & Nylon, PE, PP, PVC & $100-4000$ & 10 & 12 \\
\hline \multirow{6}{*}{ Atlantic Ocean } & Brazil & PET, PP & $100-1000$ & 200 & 12 \\
\hline & Portugal & PET, PP & $160-980$ & $0-10$ & 11 \\
\hline & Senegal & PE, PET, PP & $100-3000$ & 250 & 12 \\
\hline & South Africa & PET & $160-980$ & $1-3$ & 11 \\
\hline & United Kingdom & PP, PE, PVC & $100-2000$ & 120 & 12 \\
\hline & USA & PE & $100-1000$ & 3000 & 12 \\
\hline
\end{tabular}

Table 2. Comparison of present and worldwide values for microplastics abundance, size range, and polymer types in commercial sea salt samples. PET Polyethylene terephthalate, $P P$ Polypropylene, $P E$ Polyethylene, $P V C$ Polyvinyl chloride, PS Polystyrene, PA Polyamide, PU Polyurethane.

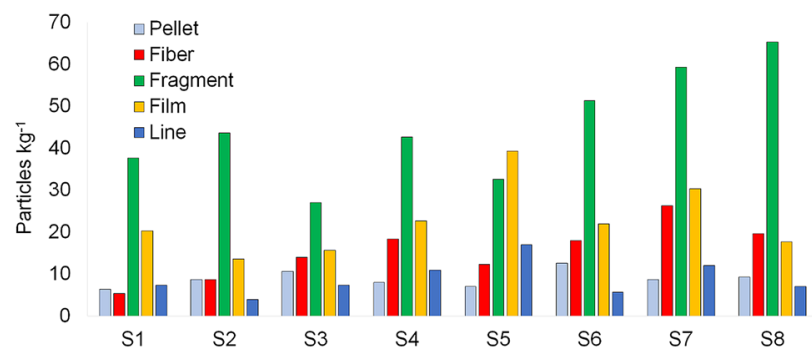

Figure 5. Microplastics abundance (particles $\mathrm{kg}^{-1}$ ) by shape category registered at the sampling sites S1 to S8.

The results obtained in this study have been compared with other salt studies worldwide (Table 2). However, it should be mentioned that most studies were developed with refined commercial sea salt samples and not from field salt pans as in this case. In addition, the different analytical methods used for MP determination difficult results comparison. The MP concentrations found in the salts from MC are similar to those reported by studies in Brazil, Mexico, South Korea, and Indonesia ${ }^{12,27}$ (Table 2). On the other hand, studies of salt samples from the Atlantic and Indian Oceans ${ }^{12}$, the Pacific Ocean (China and Thailand) ${ }^{19}$, and the Mediterranean Sea (Croatia, Italy) ${ }^{27,28}$ presented higher MP concentrations (Table 2). These studies detected the presence of MP of smaller size than those registered here, supporting the observation of the higher values. It could be expected that the fragmentation of MP particles during salt processing for commercial salts could also be contributing to the increasing number of particles found in salt samples.

MP shape, size, and color. The fragment and film MP categories were the most abundant shape types (Table 1, Fig. 5), coinciding with the results reported for sea salts samples worldwide ${ }^{12}$. The order of distribution based on MP shape was: fragments $(48 \%)>$ films $(22 \%)>$ fibers $(15 \%)>$ pellets and lines (both $9 \%)$. Higher quantities of fragments and films were also reported for Indian salts samples ${ }^{20}$. The studies analyzing MP in Cox's 


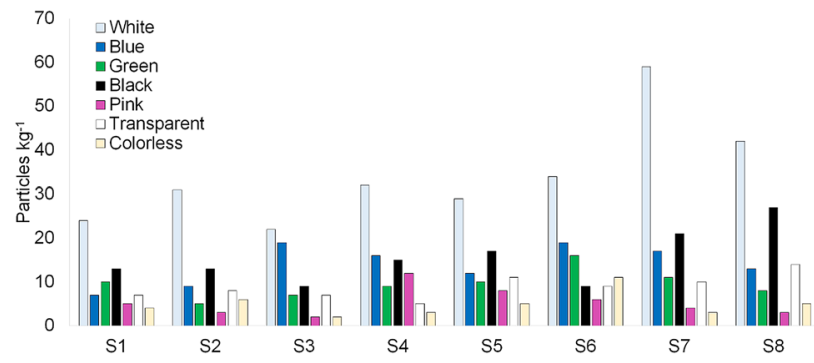

Figure 6. Microplastics abundance (particles $\mathrm{kg}^{-1}$ ) by color in sea salt samples from stations S1 to S8.

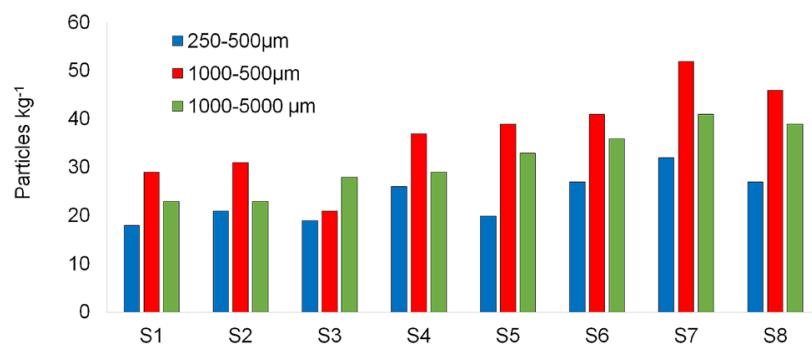

Figure 7. Microplastics abundance (particles $\mathrm{kg}^{-1}$ ) by size range in sea salt samples from stations S1 to S8.

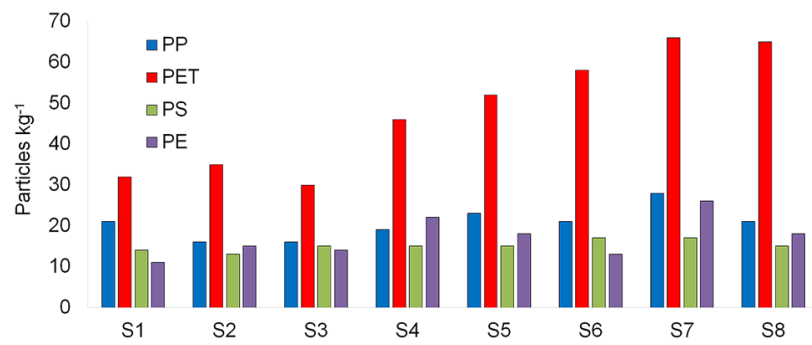

Figure 8. Microplastics abundances (particles $\mathrm{kg}^{-1}$ ) by polymer composition in sea salt samples from stations S1 to S8.

Bazar sediments registered fibers and fragments dominating shape composition ${ }^{15,23-26}$. Given that this area is highly touristic and urbanized, plastic fibers from clothes and fabrics could be a major source.

The abundance of MP in salt samples by color and size range is presented in Figs. 6 and 7 and Table 1. The colors identified include white, blue, green, black, pink, transparent, and colorless. The distribution was: white $(37 \%)>$ black $(17 \%)>$ blue $(15 \%)>$ green and transparent $(10 \%$ each $)>$ pink $(6 \%)>$ colorless $(5 \%)$. In terms of size, most particles were in the category 500-1000 $\mu \mathrm{m}$, except for S3 (1000-5000 $\mu \mathrm{m})$ (Table 1). The distribution of MP particles based on size range was: $500-1000 \mu \mathrm{m}(40 \%)>1000-5000 \mu \mathrm{m}(34 \%)>250-500 \mu \mathrm{m}(26 \%)$. For salts from the Atlantic and the Pacific Ocean, originating from Brazil, the United Kingdom, and the USA, Kim et al. ${ }^{12}$ reported a higher abundance of MP in size range $100-1000 \mu \mathrm{m}$ while sizes in the range 100-5000 $\mu \mathrm{m}$ were reported for salt samples from the Black Sea. Seth and Shriwastay ${ }^{20}$ found that $80 \%$ of fibers found in salt samples from the Indian Sea were smaller than $2000 \mu \mathrm{m}$ in length. MP size range differences among the various studies are suggested to depend on the degree of weathering for a given environment ${ }^{30}$, different climatic conditions such as wind, rain, temperature, salinity, and waves influencing size range composition. Also, for runoff, rivers, and atmospheric fallout transportation, smaller MP size ranges can be expected to be associated with a longer range from the initial plastic sources ${ }^{31-33}$. Nevertheless, more detailed information about MP polymer/color features within the size ranges are needed to achieve stronger conclusions about potential long/short-range sources.

MP polymer composition. Four types of polymer, namely polypropylene (PP), polystyrene (PS), polyethylene (PE), and polyethylene terephthalate (PET), were identified with FT-MIR-NIR (Supplementary Figure S1). These results are in accordance with those reported for salt samples in other studies worldwide (Table 1). These polymer types are widely used in daily life products, packaging, single-use plastics, and clothes, contributing to plastic pollution worldwide ${ }^{21}$. PET presented the highest contribution at all sampling sites, at $\sim 48 \%$, whereas PS was found to be least, at $\sim 15 \%$ (Fig. 8, Table 1). Iñiguez et al..$^{34}$ also reported PET predominance $(83.3 \%$ ) 


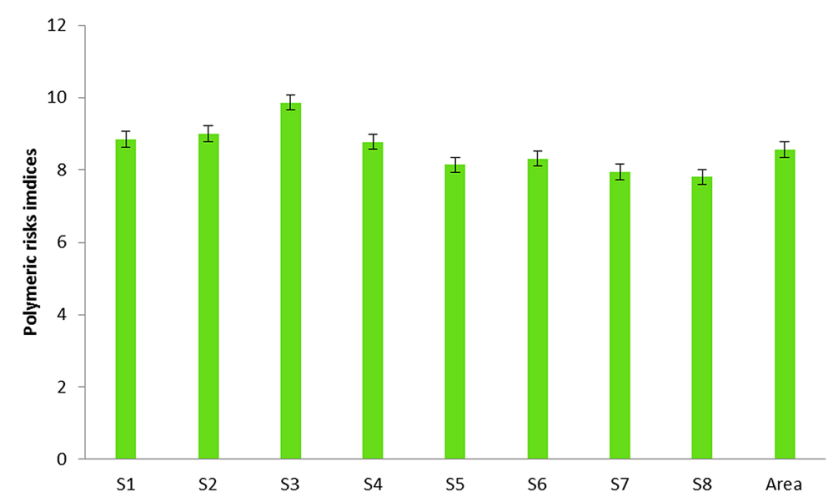

Figure 9. Polymeric risk indices for MP types in salts from stations S1 to S8.

in Spanish table salt samples. PET predominance could be explained by its high density $\left(1.30 \mathrm{~g} \mathrm{~cm}^{-3}\right)$, making particles prone to sedimentation during the salt crystallization process ${ }^{19}$. $\mathrm{PE}\left(0.94 \mathrm{~g} \mathrm{~cm}^{-3}\right), \mathrm{PP}\left(0.90 \mathrm{~g} \mathrm{~cm}^{-3}\right)$, and PS $\left(1.05 \mathrm{~cm}^{-3}\right)$ presented lower or similar densities to seawater $\left(\sim 1.02 \mathrm{~g} \mathrm{~cm}^{-3}\right)$, making these more prone to flotation and possible loss due to wind during desiccation.

Risks assessment. During degradation, MP tends to emit monomers and different types of additives, these having the potential to cause harm to ecological systems and health ${ }^{18,35}$. Results for the polymeric risks indices are presented in Fig. 9. According to polymer risk classification, all salts samples showed low risks, being similar to the entire study area. To date, none of the published studies have applied chemometric models in evaluating MP pollution in salts, posing difficulties when comparing our results. Information on the hazards of MP from ingestion to human health is still highly unclear. Other than exposure, the destiny and transit of ingested MP in the human body, including intestinal digestion and biliary discharge, have not been determined in previous research and remained largely unclear ${ }^{36}$. Some studies conducted impact assessments based on in vitro models $s^{37,38}$. However, whether the exposure concentrations used in such studies indicate the MP consumed and collected in humans is inconclusive. Previous studies found that toxicity, oxidative stress, and inflammation could result from MP exposure, including immune disruption and neurotoxicity effects, among others ${ }^{39}$. Therefore, an immediate effort is required to assess the health consequences of these MP when they reach the human body.

\section{Conclusions}

Microplastics in salt have become a critical issue of environmental pollution and public health. The present study has provided the first report on MP contamination in coarse salt samples from Bangladesh. A total of eight salt pans along the Maheshkhali Channel of the Bay of Bengal were selected for this study, all samples showing the presence of MP concentrations in the range from $78 \pm 9.33$ to $137 \pm 21.70$ particles $\mathrm{kg}^{-1}$. Most MPs were white and in the size range $500-1000 \mu \mathrm{m}$. The predominant shapes were fragments and films (70\%). Fourier transform midIR spectrum, and near-IR spectrum (FT-MIR-NIR) analysis registered the presence of polyethylene terephthalate (48\%), polypropylene (20\%), polyethylene (17\%), and polystyrene (15\%) in all samples. The plastic cover used for salt desiccation, urbanization including household effluents, local fisheries, industries, and tourism activities, were the primary potential plastic pollution sources. The results contribute to a better knowledge of MP presence in sea salts in Bangladesh and may help to prompt actions to reduce human exposure to MP in the future.

Received: 14 September 2021; Accepted: 10 November 2021

Published online: 30 November 2021

\section{References}

1. Isobe, A., Iwasaki, S., Uchida, K. \& Tokai, T. Abundance of non-conservative microplastics in the upper ocean from 1957 to 2066. Nat. Commun. 10, 1-3. https://doi.org/10.1038/s41467-019-08316-9 (2019).

2. Gewert, B., Plassmann, M. M. \& Macleod, M. Pathways for degradation of plastic polymers floating in the marine environment. Environ. Sci. Process. Impacts 17(9), 1513-1521 (2015).

3. Blair, R. M., Waldron, S., Phoenix, V. \& Gauchotte-Lindsay, C. Micro- and nanoplastic pollution of freshwater and wastewater treatment systems. Springer Sci. Rev. 5(1-2), 19-30 (2017).

4. Jambeck, W., Geyer, J. R., Siegler, T. R., Andrady, K. M. \& Law, A. Plastic waste inputs from land into the ocean. Science 347(6223), 768-771 (2015).

5. Cole, M. et al. Isolation of microplastics in biota-rich seawater samples and marine organisms. Sci. Rep. 4, 1-8. https://doi.org/10. 1038/srep04528 (2014).

6. Mauludy, M. S., Yunanto, A. \& Yona, D. Microplastic abundances in the sediment of coastal beaches in Badung. Bali. J. Perikan. Univ. Gadjah Mada 21(2), 73 (2019).

7. Storck, F. R., Kools, S. A. \& Rinck-Pfeiffer, S. Microplastics in Fresh Water Resources (Global Water Research Coalition, Stirling, 2015).

8. Zhang, W. et al. Microplastic pollution in the surface waters of the Bohai Sea, China. Environ. Pollut. 231, 541-548 (2017). 
9. Shahadat, H., Kwei, M., Lin, C. Land Use Zoning for Integrated Coastal Zone Management Remote Sensing, GIS and RRA Approach in Cox's Bazar Coast, Bangladesh. Bangladesh. ITCZM Monogr (2001).

10. Serrano, R., Nacher-Mestre, J., Portoles, T., Amat, F. \& Hernandez, F. Non-target screening of organic contaminants in marine salts by gas chromatography coupled to high-resolution time of flight mass spectrometry. Talanta 85, 877-884 (2011).

11. Karami, A. et al. The presence of microplastics in commercial salts from different countries. Sci. Rep. 7(1), 1-11 (2017).

12. Kim, J. S., Lee, H. J., Kim, S. K. \& Kim, H. J. Global pattern of microplastics (MPs) in commercial food-grade salts: Sea salt as an indicator of seawater MP pollution. Environ. Sci. Technol. 52(21), 12819-12828 (2018).

13. Barua, D. K. Dynamics of coastal circulation and sediment transport in the coastal ocean off the Ganges-Brahmaputra river mouth (Doctoral dissertation, University of South Carolina) (1992).

14. Aktar, M., Azam, M. A. K. \& Siddique, M. A. M. Trace metal concentrations in the green-lipped mussel perna viridis (Linnaeus, 1758) collected from Maheshkhali channel, Cox's Bazar. Bangladesh. J. Fish. https://doi.org/10.3153/jfscom.2014005 (2014).

15. Rakib, M. R. J., De-la-Torre, G. E., Pizarro-Ortega, C. I., Dioses-Salinas, D. C. \& Al-Nahian, S. Personal protective equipment (PPE) pollution driven by the COVID-19 pandemic in Cox's Bazar, the longest natural beach in the world. Mar. Pollut. Bull. 2, 112497 (2021).

16. Misra, S. K., Chandramohan, P., Satyanarayana, M. A., Panigrahi, J. K. \& Mahadevan, R. Nature of the tide induced flow field along the East Coast of India. Int. J. Oceans Oceanogr 7(1), 57-71 (2013).

17. Dunn, O. J. Multiple comparisons among means. J. Am. Stat. Assoc. 56(293), 52-64 (1961).

18. Enyoh, C. E., Verla, A. W. \& Rakib, M. R. J. Application of index models for assessing freshwater microplastics pollution. World News Nat. Sci. 38, 37-48 (2021).

19. Yang, D. et al. Microplastic pollution in table salts from China. Environ. Sci. Technol. 49(22), 13622-13627 (2015).

20. Seth, C. K. \& Shriwastav, A. Contamination of Indian sea salts with microplastics and a potential prevention strategy. Environ. Sci. Pollut. Res. 25(30), 30122-30131 (2018).

21. Enyoh, C. E., Verla, A. W., Verla, E. N., Ibe, F. C. \& Amaobi, C. E. Airborne microplastics: A review study on method for analysis, occurrence, movement and risks. Environ. Monit. Assess. 191, 668. https://doi.org/10.1007/s10661-019-7842-0 (2019).

22. Enyoh, C. E. et al. Microplastics exposure routes and toxicity studies to ecosystems: An overview. EAHT 35(1), 1-10. https://doi. org/10.5620/eaht.e2020004 (2020).

23. Alfonso, M. B., Arias, A. H., Ronda, A. C. \& Piccolo, M. C. Continental microplastics: Presence, features, and environmental transport pathways. Sci. Total Environ. 799, 149447 (2021).

24. Rahman, S. M. A., Robin, G. S., Momotaj, M., Uddin, J. \& Siddique, M. A. M. Occurrence and spatial distribution of microplastics in beach sediments of Cox's Bazar. Bangladesh. Mar. Pollut. Bull. 160, 111587. https://doi.org/10.1016/j.marpolbul.2020.111587 (2020).

25. Hossain, M. B., Banik, P., Nur, A. A. U. \& Rahman, T. Abundance and characteristics of microplastics in sediments from the world's longest natural beach, Cox's Bazar, Bangaladesh. Mar. Pollut. Bull. 163, 111956 (2021).

26. Tajwar, M., Gazi, M. Y. \& Saha, S. K. Characterization and spatial abundance of microplastics in the coastal regions of Cox's Bazar, Bangladesh: An integration of field, laboratory, and GIS techniques. Soil Sediment. Contam. 00, 1-25. https://doi.org/10.1080/ 15320383.2021.1910622 (2021).

27. Kosuth, M., Mason, S. A. \& Wattenberg, E. V. Anthropogenic contamination of tap water, beer, and sea salt. PLoS ONE 13(4), e0194970 (2018).

28. Renzi, M. \& Blašković, A. Litter \& microplastics features in table salts from marine origin: Italian versus Croatian brands. Mar. pollut. Bull. 135, 62-68 (2018).

29. Gündoğdu, S. Contamination of table salts from Turkey with microplastics. Food Addit. Contam. Part A 35(5), 1006-1014 (2018).

30. Alfonso, M. B., Ronda, A. C., Piccolo, M. C. \& Arias, A. H. Microplastic pollution: From continental sources to marine systems Contributor Copy. In Marine Environments: Diversity (ed. Charles, L.) 305-339 (Nova Science Publishers, 2021).

31. Brahney, J., Hallerud, M., Heim, E., Hahnenberger, M. \& Sukumaran, S. Plastic rain in protected areas of the United States. Science 368, 1257-1260. https://doi.org/10.1126/science.aaz5819 (2020).

32. Evangeliou, N. et al. Atmospheric transport is a major pathway of microplastics to remote regions. Nat. Commun. https://doi.org/ 10.1038/s41467-020-17201-9 (2020).

33. Weideman, E. A., Perold, V., Omardien, A., Smyth, L. K. \& Ryan, P. G. Quantifying temporal trends in anthropogenic litter in a rocky intertidal habitat. Mar. Pollut. Bull. 160, 111543 (2020).

34. Iñiguez, M. E., Conesa, J. A. \& Fullana, A. Microplastics in Spanish table salt. Sci. Rep. 7(1), 1-7 (2017).

35. Yee, M. S. L. et al. Impact of microplastics and nanoplastics on human health. Nanomaterials 11, 496. https://doi.org/10.3390/ nano11020496 (2021).

36. Ibrahim, Y. S. et al. Detection of microplastics in human colectomy specimens. JGH Open 5, 116-121 (2021).

37. Liao, Y. L. \& Yang, J. Y. Microplastic serves as a potential vector for $\mathrm{Cr}$ in an in-vitro human digestive model. Sci. Total Environ. 703, $134805(2020)$.

38. Liu, S., Wu, X., Gu, W., Yu, J. \& Wu, B. Influence of the digestive process on intestinal toxicity of polystyrene microplastics as determined by in vitro Caco-2 models. Chemosphere 256, 127204 (2020).

39. Prata, J. C., da Costa, J. P., Lopes, I., Duarte, A. C. \& Rocha-Santos, T. Environmental exposure to microplastics: An overview on possible human health effects. Sci. Total Environ. 702, 134455 (2020).

\section{Acknowledgements}

The authors gratefully acknowledge the Bangladesh Oceanographic Research Institute for providing the laboratory facilities for this study; also to Taif University Researchers Supporting Project number (TURSP-2020/163), Taif 21944, Saudi Arabia.

\section{Author contributions}

M.R.J.R.: conceptualization, methodology, validation, formal analysis, data curation, investigation, project administration, writing - original draft, writing - review and editing. S.A.-N.: methodology, validation, formal analysis, software, investigation. M.B.A.: writing-original draft, formal analysis, investigation. M.U.K., C.E.E., S.H.F., A.A., A.S.A.A., D.A.B., H.M., M.A.I.: writing-review and editing.

\section{Competing interests}

The authors declare no competing interests.

\section{Additional information}

Supplementary Information The online version contains supplementary material available at https://doi.org/ 10.1038/s41598-021-02457-y. 
Correspondence and requests for materials should be addressed to M.J.R. or H.M.

Reprints and permissions information is available at www.nature.com/reprints.

Publisher's note Springer Nature remains neutral with regard to jurisdictional claims in published maps and institutional affiliations.

(c) (i) Open Access This article is licensed under a Creative Commons Attribution 4.0 International cc) License, which permits use, sharing, adaptation, distribution and reproduction in any medium or format, as long as you give appropriate credit to the original author(s) and the source, provide a link to the Creative Commons licence, and indicate if changes were made. The images or other third party material in this article are included in the article's Creative Commons licence, unless indicated otherwise in a credit line to the material. If material is not included in the article's Creative Commons licence and your intended use is not permitted by statutory regulation or exceeds the permitted use, you will need to obtain permission directly from the copyright holder. To view a copy of this licence, visit http://creativecommons.org/licenses/by/4.0/.

(C) The Author(s) 2021 